Notas técnicas 



\title{
DISEÑO Y CONSTRUCCIÓN DE CELDAS DE CARGA PARA COMPROBACIONES INTERMEDIAS DE MÁQUINAS DE FUERZA
}

\section{Resumen}

En este artículo se presenta una metodología para el diseño y la construcción de celdas de carga de geometría sencilla y de bajo costo que funcionan en compresión, para ser utilizadas en la comprobación (también llamada verificación) intermedia de máquinas de ensayo donde interviene la magnitud fuerza y de uso común en los laboratorios de materiales de construcción para obras civiles.

Estas celdas de carga se convierten en una herramienta muy útil para los laboratorios que operan bajo un sistema de calidad basado en la norma INTE ISO/IEC 17025:2005 para determinar si las máquinas de ensayo que utiliza están brindando resultados de fuerza correctos entre periodos de calibración o cuando el usuario tiene dudas de sus resultados.

Palabras clave: Celda, fuerza, galgas, comprobación, laboratorios.

\begin{abstract}
This article presents a methodology for design and manufacture of compression load cells of simple geometry and low cost, to be used to verify testing machines commonly used in civil engineering materials testing laboratories.

These load cells are useful instruments for laboratories that operate under INTE ISO/IEC 17025:2005 quality systems, to determine if the testing machines are displaying correct force readings between scheduled calibrations or when the user has doubts about the results.
\end{abstract}

Keywords: Design, load cell, force, calibrations, laboratories.

Recibido: 27 de Abril del 2011 • Aprobado: 15 de Mayo del 2012

\section{INTRODUCCIÓN}

Las celdas de carga son instrumentos de medición de fuerza que cada vez se utilizan más en la industria, la ingeniería, por universidades en investigación, en los laboratorios de ensayo para el control de calidad de materiales en general, entre otros. Por lo tanto, el objetivo de este artículo es presentar una metodología para el diseño y la construcción de celdas de carga de geometría sencilla y de bajo costo que funcionan en compresión, para ser utilizadas en la comprobación (también llamada verificación) intermedia de máquinas de fuerza uniaxial de uso común en los laboratorios de materiales de construcción para obras civiles. También se presenta como ejemplo, la construcción de una celda de carga, para ilustrar la metodología empleada.

Las celdas de carga son básicamente transductores, es decir, dispositivos que convierten energía mecánica en señales eléctricas. El término celda es utilizado de forma conveniente para describir un transductor compacto. Las celdas de carga son instrumentos electrónicos que miden fuerza (carga), utilizando como principio la Ley de Hooke. Este principio aplicado en la celda de carga aprovecha las propiedades elásticas de un material, en este caso el acero y mediante el uso de galgas extensométricas (strain gages), se mide la deformación unitaria del elemento elástico y se relaciona con el valor de la fuerza aplicada sobre él. Existen 
celdas de carga de tracción, compresión y universales (compresión y tracción).

En el Laboratorio Nacional de Materiales y Modelos Estructurales de la Universidad de Costa Rica (LanammeUCR), se han fabricado celdas de carga de compresión que se han utilizado en ensayos de laboratorio rutinarios y que han funcionado durante años satisfactoriamente. Este conocimiento del LanammeUCR se convierte en la base para establecer un procedimiento de diseño y fabricación de celdas de carga sencillos, desde el punto de vista de su aplicación.

El procedimiento antes mencionado se aprovecha en la fabricación de celdas de carga que pueden ser utilizadas para realizar comprobaciones intermedias en las máquinas de ensayo que se utilizan comúnmente en un laboratorio de materiales de construcción. A estas celdas de carga habitualmente se les conoce como patrones de trabajo, patrón de verificación (comprobación) o patrón de control.

El periodo de calibración recomendado para las máquinas de ensayo de fuerza uniaxial es usualmente de un año y sin sobrepasar los 18 meses (American Society for Testing and Materials, 2009). Sin embargo, este tipo de máquinas que se utilizan con una frecuencia muy alta, con varios operadores y a veces por periodos de tiempo prolongados, requieren de comprobaciones intermedias, es decir, revisiones no tan exhaustivas como las calibraciones, pero que demuestren que la máquina está reportando valores de carga confiables. Lo anterior también aplica si se realizan reparaciones o se reubica la máquina. De ahí la necesidad de contar a lo interno de un laboratorio con un instrumento de medición de fuerza (patrón de comprobación) para hacer comprobaciones intermedias periódicas de las máquinas de ensayos a intervalos más cortos que los normalmente establecidos para las calibraciones, o bien, para comprobar la exactitud de las máquinas de fuerza en el momento en que se tengan dudas de sus resultados. La comprobación intermedia entre periodos de calibración de estas máquinas de ensayo es fundamental para el aseguramiento de la calidad de los laboratorios de materiales, específicamente para demostrar la confianza de los resultados de los ensayos. Esto último es especialmente importante para aquellos laboratorios de materiales que operan siguiendo los lineamientos de la norma ISO/IEC 17025:2005 y que utilizan máquinas de fuerza.

El diseño de celda de carga que se presenta en este documento, ha demostrado un desempeño satisfactorio para instrumentos con capacidad máxima nominal igual o superior a los $100 \mathrm{kN}$ (10 $000 \mathrm{~kg}_{f}$ aproximadamente). La capacidad máxima de las celdas de carga que se recomienda diseñar está limitada por la capacidad máxima (alcance) del laboratorio de calibración de la magnitud fuerza de nuestro país.

Para efectos prácticos, una celda de carga con una capacidad nominal de $1000 \mathrm{kN}$ es idónea. Lo anterior, se fundamenta en el hecho de que la mayoría de los laboratorios de materiales de construcción utilizan máquinas para ensayar de cilindros de concreto a compresión, cuya capacidad ronda los $1000 \mathrm{kN}$, sumado al hecho de que en el país se pueden calibrar celdas de carga de esa capacidad.

La evaluación de la idoneidad de las celdas de carga construidas depende de los resultados de su calibración. Dicha calibración debe realizarse con métodos y patrones de fuerza adecuados y su resultado debe demostrar niveles de precisión y exactitud acordes con el fin propuesto(ISO 10012, 2003).

Es importante dejar claro que el instrumento de medición de fuerza que se presenta en este documento tiene como fin servir como referencia para la comprobación intermedia de máquinas de ensayo (patrón de comprobación). En principio, su uso para realizar calibraciones no está recomendado, a no ser que los resultados de su calibración demuestren que el instrumento propuesto alcanza los niveles de precisión y exactitud requeridos para calibrar, los cuales usualmente son mucho mayores que los de un instrumento de referencia para comprobaciones intermedias o de trabajo.

\section{PRINCIPIO DE FUNCIONAMIENTO DE UNA CELDA DE CARGA}

"Es frecuente emplear elementos elásticos a fin de indicar la magnitud de una fuerza aplicada midiendo el desplazamiento" (Holman, s.f.) o bien, su deformación (Ley de Hooke). La celda de carga es un dispositivo que se utiliza para la medición de fuerza, que está compuesto por 
un elemento con propiedades elásticas que se ha instrumentado con galgas extensométricas (strain gages), para brindar información exacta y precisa sobre la magnitud de la fuerza aplicada sobre él, a partir de su deformación unitaria. La galga extensométrica consiste en una lámina de un material a la cual se le ha impreso un circuito eléctrico resistivo. La galga se adhiere firmemente al elemento sometido a cargas de tracción o compresión. Al aplicar carga sobre el elemento con la galga adherida, esta última registra un cambio en su resistencia que se asocia a la deformación unitaria del elemento. El valor de deformación unitaria está directamente asociado con la carga aplicada.

Desde el punto de vista de instrumentación, es necesario colocar 4 galgas extensométricas sobre la parte central del cuerpo de la celda de carga, y realizar conexiones eléctricas de manera que se forme un circuito conocido como Puente de Wheatstone, el cual permite contrarrestar esfuerzos no deseados (BLH Electronics, s.f.). En la Figura 1 se muestra el esquema del Puente de Wheatstone y la forma en que se colocan las galgas extensométricas sobre el cuerpo de la celda de carga.

Para un arreglo como el que se muestra en la Figura 1, el puente de Wheatstone funciona de la siguiente forma: las galgas extensométricas R1 y R2 miden la deformación axial. Si se presenta flexión, sus efectos en las galgas R1 y R2 serán iguales pero de signos opuestos, lo cual los cancela. Las galgas R3 y R4, van a eliminar los efectos ocasionados por la variación de temperatura.

Entre las ventajas del Puente de Wheatstone debidas a su forma de operar se tienen:

- Mide las deformaciones unitarias con mucha precisión y exactitud.

- Es muy sensible, lo cual es deseable para detectar deformaciones unitarias muy pequeñas, del orden de 1 x 10-6 (Harris, s.f).

- Hay un efecto propio del circuito de autocompensación por temperatura en un rango que usualmente se encuentra $18^{\circ} \mathrm{C}$ y los $35^{\circ} \mathrm{C}$ aproximadamente, lo cual le permite trabajar adecuadamente sin tener que hacer correcciones por este
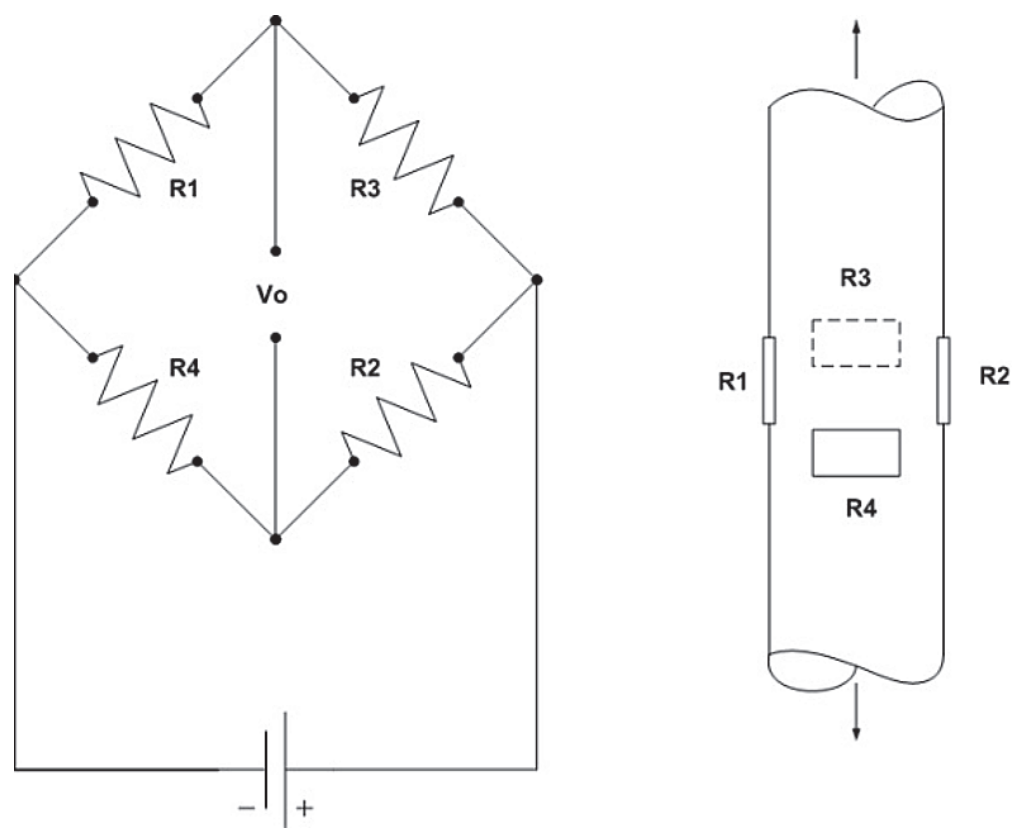

Figura 1. Diagrama de un puente de Wheatstone y su arreglo con galgas extensométricas sobre el cuerpo de la celda de carga. La galga $R_{3}$ está ubicada diametralmente opuesta a la galga $R_{4}$. 
efecto. Esta propiedad es válida siempre y cuando las variaciones de temperatura durante su utilización sean muy graduales.

4- Se pueden compensar esfuerzos no deseado, al cancelarse sus efectos según se explicó en el caso de la flexión, producto de alguna excentricidad al aplicar la carga (BLH Electronics, s.f.).

\section{MATERIALES PARA LA CELDA DE CARGA}

La selección de los materiales para la construcción de cualquier celda de carga en general, es determinante para su buena operación. De nada vale realizar un buen diseño, si los materiales utilizados para su construcción no cumplen con ciertos requisitos mínimos.

El acero es el primer material que se escoge para diseñar y luego construir una celda de carga. El acero se utiliza para el cuerpo de la celda de carga, componente principal. Específicamente en este caso se utilizó acero AISI 1020. Si bien es cierto, esta clase de acero no es común en la fabricación de celdas de carga, la experiencia ha demostrado que tiene un buen desempeño en celdas de trabajo, aparte de que es de bajo costo y fácil de maquinar.

La característica más deseable del acero es su comportamiento prácticamente lineal en el rango elástico, lo que hace que sea ampliamente utilizado para la fabricación de celdas de carga. Se pueden utilizar otros materiales como el aluminio, sin embargo, su comportamiento en el rango elástico no es lineal, lo cual dificulta el proceso.

El cobertor es un componente de la celda de carga, el cual consiste en un cilindro hueco, que se ubica alrededor del cuerpo de la celda de carga, cuya misión es proteger los componentes internos y conexiones eléctricas, una vez terminada la celda. Este cobertor también se puede confeccionar en acero AISI 1020, o bien, como no está sometido a ningún tipo de esfuerzo, se podría utilizar cualquier otro

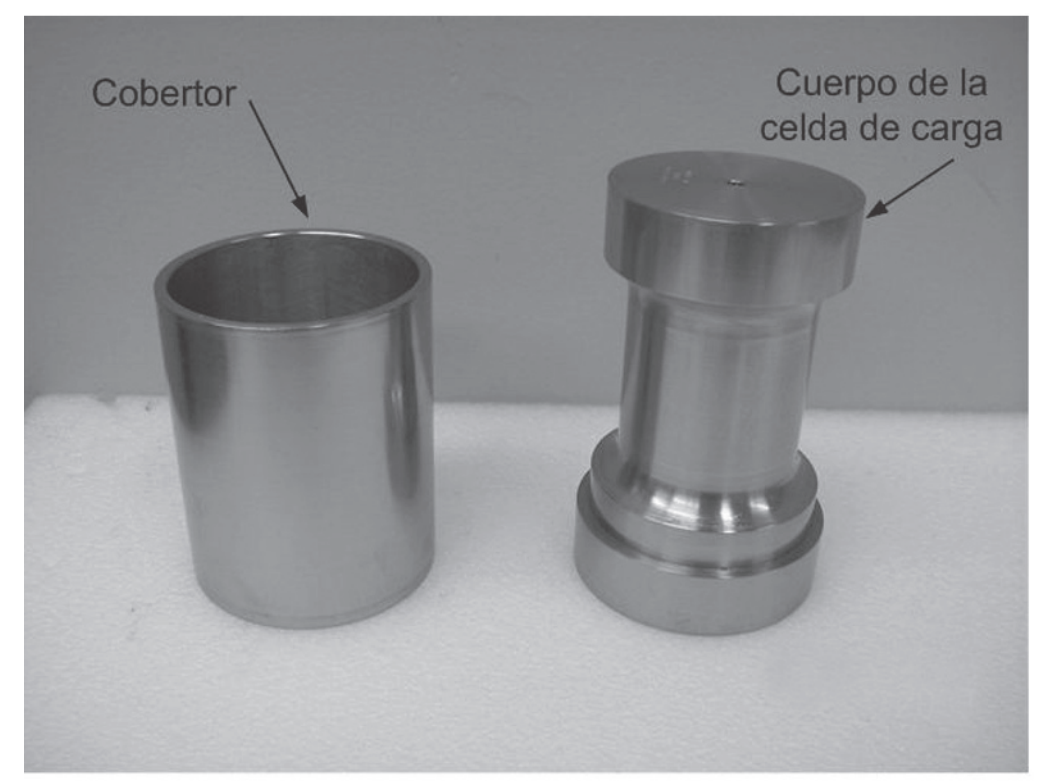

Figura 2. Cuerpo de celda con una capacidad nominal de $1000 \mathrm{kN}$ listo para ser instrumentado, con su correspondiente cobertor. Ambos se fabricaron en acero 1020. 


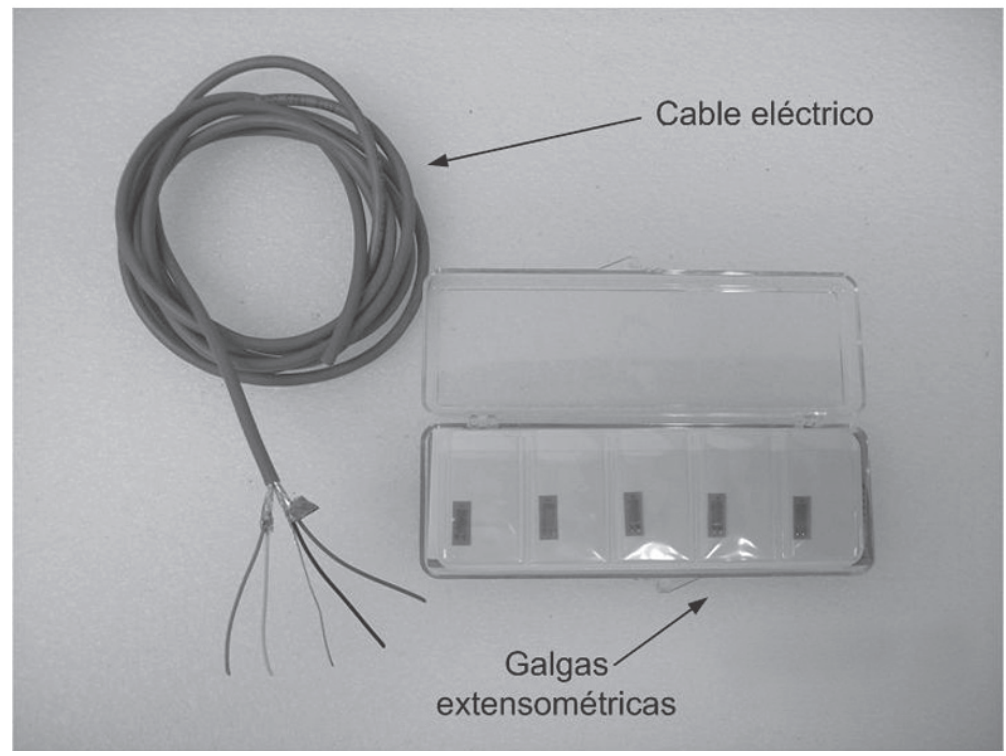

Figura 3. Muestra de galgas extensométricas y conductor eléctrico utilizados en la construcción de celdas de carga.

Fuente: Los autores.

material que cumpla su función de protección, por ejemplo, tubo de PVC.

En la Figura 2 se muestra un ejemplo del cuerpo de una celda de carga y su cobertor.

Los otros componentes internos que deben ser tomados en consideración son las galgas extensométricas y el pegamento. Deben preferirse galgas extensométricas que estén diseñadas para utilizarse sobre acero y que posean la característica de autocompensación por temperatura. Este tipo de galgas extensométricas poseen coeficientes por expansión térmica similares al del acero. Ante un cambio de temperatura, la expansión térmica del cuerpo de la celda de carga es similar a la expansión térmica de las galgas extensométricas, lo cual nos asegura que el instrumento no esté reportando lecturas erróneas por deformaciones que no sean originadas por cargas las aplicadas. Para la selección de las galgas extensométricas también debe escogerse un tamaño adecuado, de forma que sean lo suficientemente pequeñas para ser pegadas cómodamente sobre el cuerpo de la celda de carga y lo suficientemente grandes para que permitan soldar cómodamente los cables en sus terminales. Las galgas con una longitud superior a los $6 \mathrm{~mm}$ son ideales.

La selección del pegamento para las galgas extensométricas también juega un papel importante. Los pegamentos para galgas extensométricas basados en cianoacrilato y epóxicos se han utilizado con resultados satisfactorios. Usualmente los catálogos de fabricantes de galgas extensométricas brindan guías para su selección y la del pegamento, en función de la aplicación seleccionada.

Por último, no se debe olvidar el cable eléctrico. Este se encarga de transportar las señales eléctricas desde las galgas extensométricas hacia la unidad lectora. Este cable debe estar compuesto de al menos 4 conductores trensados y blindado para evitar la inducción de corrientes no deseadas o ruidos que puedan afectar las mediciones una vez que esté funcionando. Es deseable que el cable cuente con un quinto conductor, que se encargue de hacer tierra entre la celda y la caja lectora. En la Figura 3 se puede apreciar una muestra del cable y las galgas extensométricas que usualmente se utiliza para la fabricación de celdas de carga. 


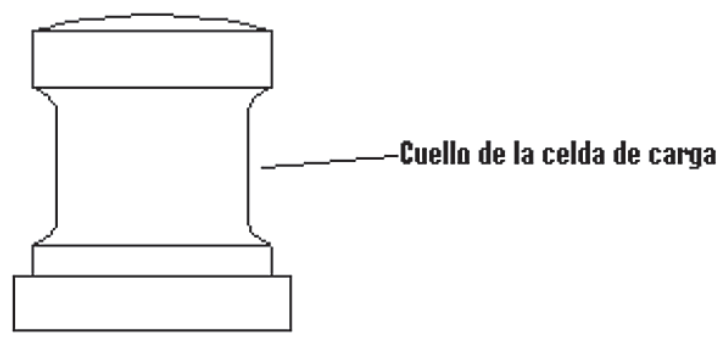

Figura 4. Forma básica del elemento elástico de una celda de carga para compresión.

Fuente: Los autores.

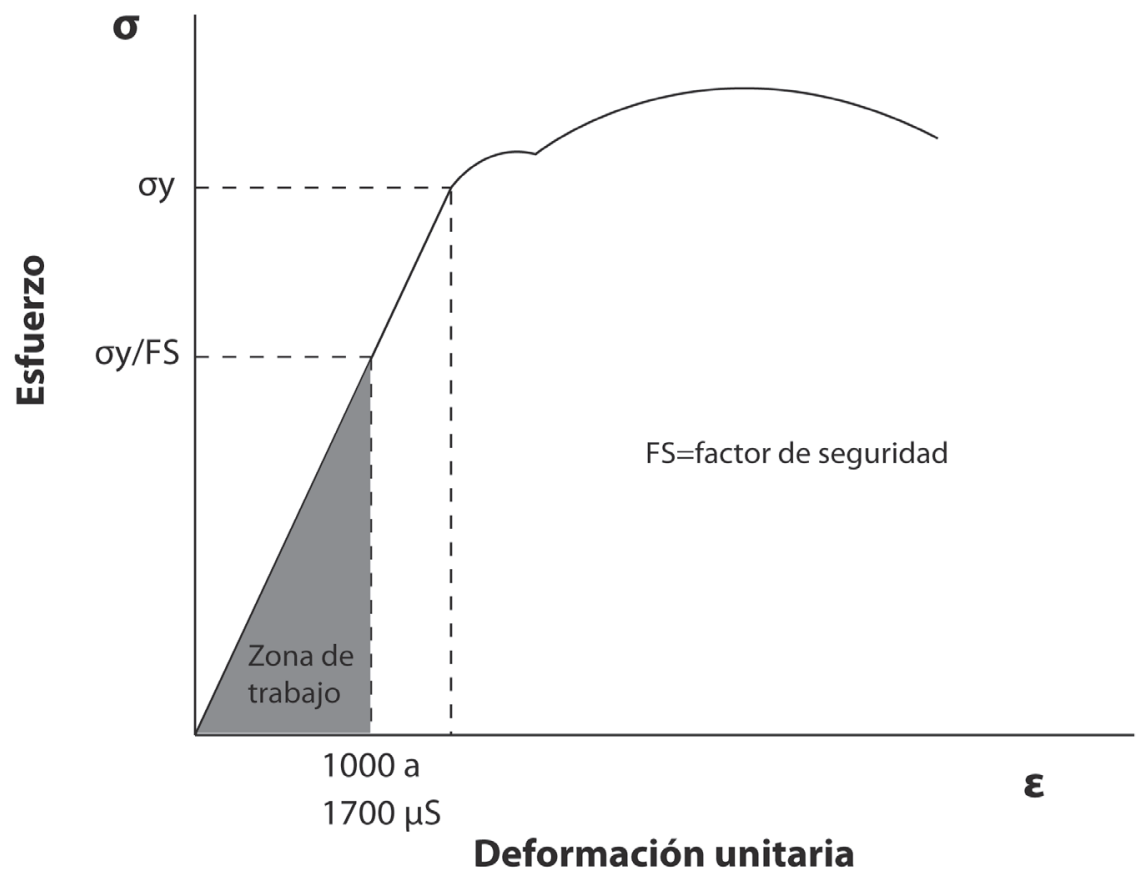

Figura 5. Gráfico del esfuerzo en función de la deformación unitaria del acero, donde se ilustra la zona de trabajo según el factor de seguridad seleccionado.

Fuente: Los autores. 


\section{DISEÑO DE LA CELDA DE CARGA}

Con respecto al diseño, se parte de una forma básica del cuerpo de la celda de carga, cuya apariencia se muestra en la Figura 4.

La forma básica y las dimensiones del elemento elástico de la celda (cuerpo), excepto el diámetro del cuello, el cual corresponde con la parte más estrecha de la Figura 4, se basan en de celdas de carga previamente construidas que han mostrado un excelente comportamiento y a la comodidad de su tamaño. El diámetro del cuello debe ser calculado para cada caso de acuerdo con la capacidad nominal deseada. La altura de la celda de carga puede ser variada en función de su aplicación. Sin embargo, no se recomienda sobrepasar las alturas recomendadas en la norma ISO 376 (2004), para minimizar los efectos por pandeo.

Para que la celda de carga funcione adecuadamente a través del tiempo, se aprovechan las propiedades elásticas del acero, en este caso AISI 1020. Se desea que la celda de carga funcione siempre en el rango elástico del material. Para asegurar esta condición, se debe considerar un factor de seguridad que debe rondar entre un $10 \%$ y un 50\% (Measurements Group, 1988). En la Figura 2 se presenta un diagrama esfuerzo deformación unitaria típico para un acero, en el cual se indica la zona de trabajo de la celda de carga de acuerdo con el factor de seguridad seleccionado.

Para efectos del diseño, lo que interesa es determinar el diámetro del cuello de la celda de carga, en función de su capacidad nominal considerando un factor de seguridad.

Este diámetro se determina mediante el uso de la ecuación básica para el cálculo de esfuerzos, $\sigma$, de mecánica de materiales:

$$
\sigma=\frac{F}{A}
$$

Donde F es la fuerza aplicada sobre el elemento elástico y A es el área sobre la cual se aplica esta fuerza. En este caso, el esfuerzo $\sigma$ es el valor del esfuerzo de fluencia del acero AISI 1020, F corresponde a la capacidad nominal de la celda de carga multiplicada por el factor de seguridad (FS) y el área A corresponde al área de la sección transversal del cuello de la celda de carga, que tiene forma circular. De la ecuación 1 , se determina el área A, a partir de la cual se calcula el diámetro del cuello de la celda de carga mediante el uso de la ecuación 2 para el cálculo del área de un círculo:

$$
A=\pi \frac{\left(d^{2}\right)}{4}
$$

Donde A es el valor del área del cuello de la celda de carga, $\pi$ es igual a 3,1415 , y d es el valor del diámetro del cuello de la celda de carga. De esa ecuación se se despeja el valor del diámetro (d) y se obtiene la ecuación 3:

$$
d=2 \sqrt{\frac{A}{\pi}}
$$

Este valor del diámetro encontrado, es el que se va a especificar en el plano para el cuello de la celda de carga. Este valor es el más importante, pues de él depende el nivel de deformación y por lo tanto la sensibilidad de la celda de carga. Si este diámetro es muy grande, el cuello se deforma poco y la celda es poco sensible (Tioli, 2007). Si es muy pequeño, la celda se deforma mucho, lo cual deja poco margen de seguridad ante una eventual sobrecarga.

La medición de la deformación unitaria se realiza por medio de las galgas extensométricas y una unidad lectora de deformación unitaria.

La viabilidad de este proyecto presupone la disponibilidad de la unidad lectora de deformación unitaria, una unidad lectora que sea capaz de leer la respuesta en $\mathrm{mV} / \mathrm{V}$ de las señales eléctricas alimentadas, o bien, una unidad lectora o de adquisición de datos para diferentes tipos de transductores. Las unidades lectoras de deformación unitaria generalmente forman parte del equipo de medición de los laboratorios de materiales de construcción.

En la Figura 6 se muestran diferentes modelos de cajas lectoras. En la tabla 1 se hace una comparación de algunas características de estas cajas lectoras. El modelo TC-31L de la casa 
Cuadro 1. Comparación de 3 modelos de cajas lectoras para celdas de carga

\begin{tabular}{ccccc}
\hline Marca & Modelo & Voltaje de excitación & Fuente de poder & Otras características \\
\hline Vishay & P-3500 & $2 \mathrm{~V}$ & Baterías & Portátil para uso en campo. \\
Vishay & P-3800 & $\begin{array}{c}\text { Variable desde 1 V } \\
\text { a 15 V }\end{array}$ & $\begin{array}{c}\text { Corriente alterna } \\
120 \mathrm{~V}\end{array}$ & Para uso en laboratorio. \\
TML & TC-31L & & Baterías & $\begin{array}{c}\text { Portátil y muy compacta. Especial para } \\
\text { uso en campo. Posee varios canales. Se } \\
\text { puede configurar para que despliegue } \\
\text { lecturas directamente en unidades de } \\
\text { fuerza u otras. }\end{array}$ \\
\hline
\end{tabular}

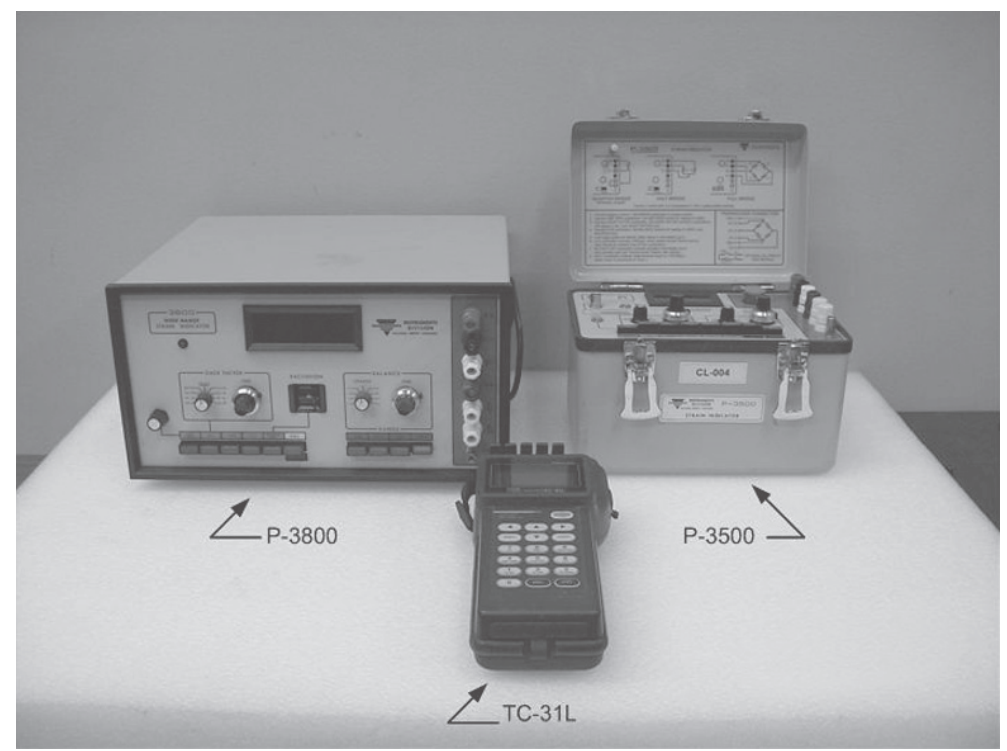

Figura 6. Unidades lectoras, modelo P-3800 de la marca Vishay, el modelo P-3500 de la marca Vishay y el modelo TC-31L de la marca TML.

Fuente: Los autores. 
TML, es muy versátil por su tamaño. Además funciona con diferentes tipos de transductores y puede desplegar lecturas en diferentes unidades de medida, lo cual resulta muy apropiado.

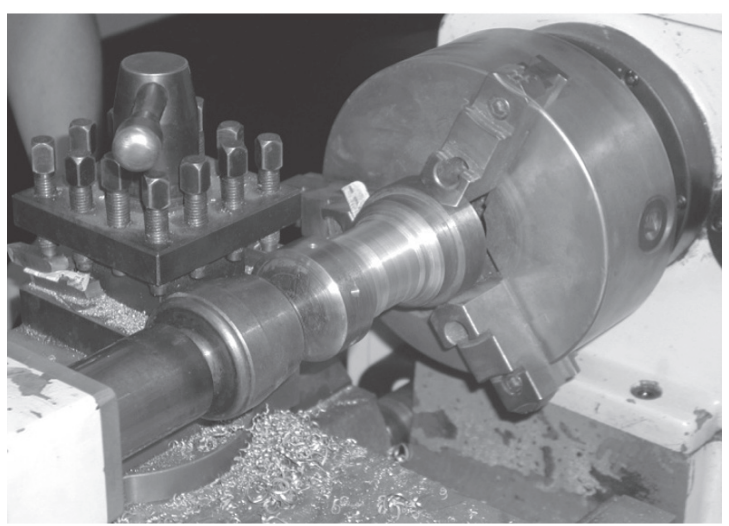

Figura 7. Maquinado de cuerpo de celda en el torno. Fuente: Los autores.

\section{FABRICACIÓN DE LA CELDA DE CARGA}

Una vez realizados los cálculos y definidas las dimensiones del cuerpo de la celda de carga, se

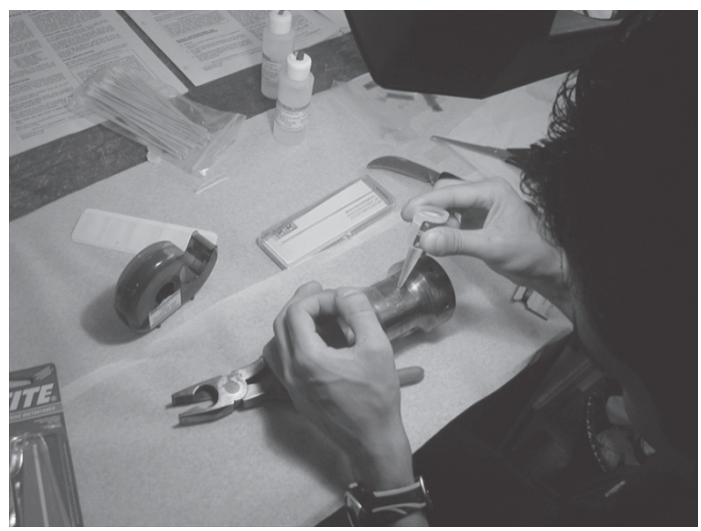

Figura 8. Instrumentando el cuerpo de la celda de carga con galgas extensométricas.

Fuente: Los autores. procede a su fabricación utilizando un torno, tal y como se muestra en la Figura 7. La dimensión principal a respetar es el diámetro del cuello.

Seguidamente se procede a instrumentar con galgas extensométricas el cuerpo de la celda de carga. Deben seguirse cuidadosamente las indicaciones dadas por el fabricante de las galgas extensométricas para no dañarlas y asegurar también una buena adherencia.

El diagrama de conexiones propuesto en la Figura 9, asegura una conexión fácil y a prueba de errores de la celda de carga con la unidad lectora. Se hacen coincidir los colores de los conductores eléctricos y los colores de las terminales de las cajas lectoras, con el fin de facilitar las conexiones y evitar errores para el usuario.

\section{PROCESO DE CALIBRACIÓN}

Una vez finalizada la construcción de la celda de carga, debe someterse a un proceso de calibración. La calibración consiste en hacer una comparación de las lecturas que ofrece la nueva celda contra un patrón y reportar los errores, es decir, las diferencias con respecto al patrón. El patrón de calibración es generalmente otra celda de carga de mayor exactitud que la que se está calibrando.

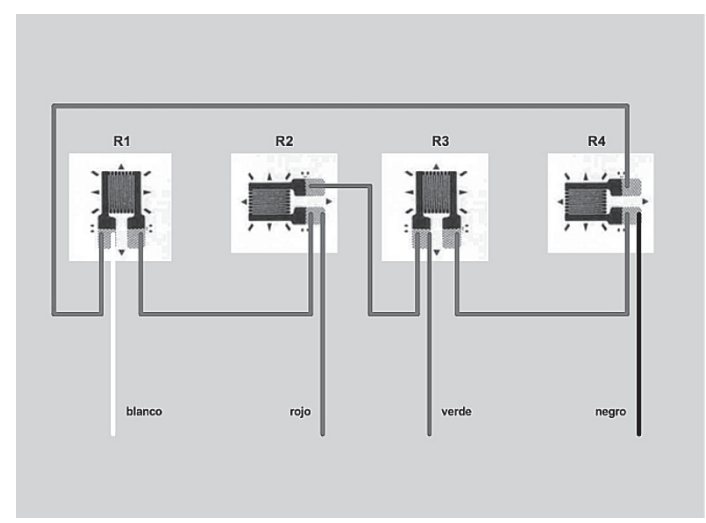

Figura 9. Diagrama de conexiones de las galgas extensométricas (Puente de Wheatstone).

Fuente: Los autores. 


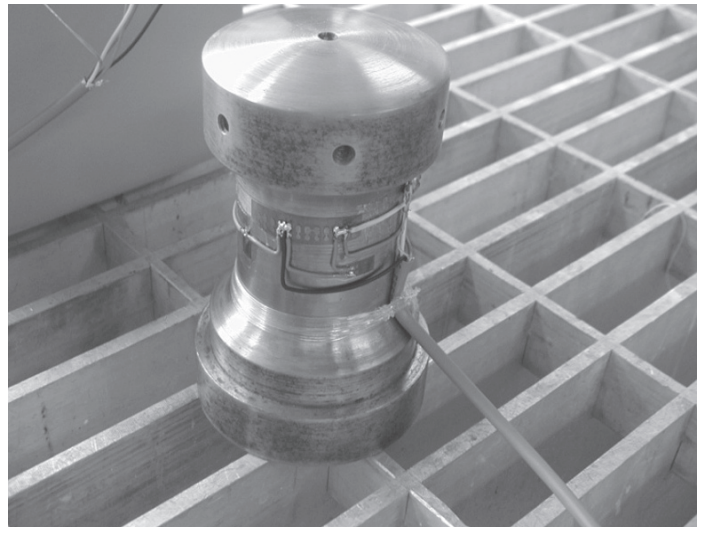

Figura 10. Cuerpo de celda de carga instrumentado. Nótense las conexiones que forman el circuito Puente de Wheatstone.

Fuente: Los autores.

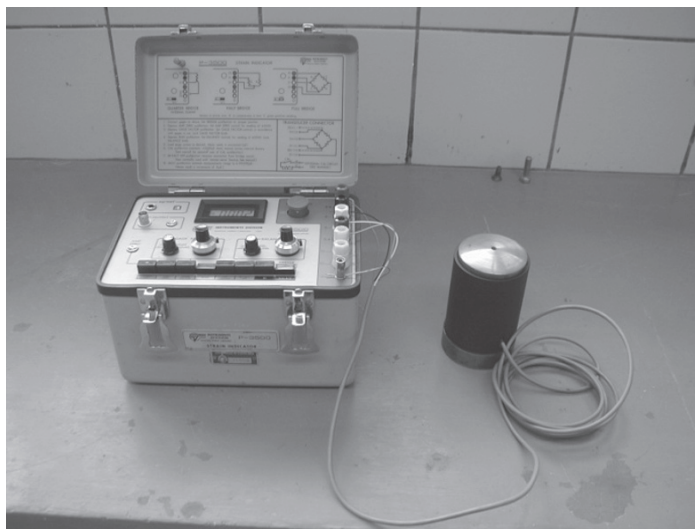

Figura 11. Celda de carga terminada y con su correspondiente cobertor, conectada en la caja lectora.

Fuente: Los autores.

Mediante la calibración, se relacionan las deformaciones medidas por la celda de carga con un valor de fuerza aplicada sobre ella. En muchos casos, dependiendo del tipo de lectora, es posible configurarla para que muestre valores de fuerza de forma directa, ya sea en kilonewton $(\mathrm{kN})$, kilogramo fuerza $\left(\mathrm{kg}_{\mathrm{f}}\right)$ o libras fuerza $\left(\mathrm{lb}_{\mathrm{f}}\right)$.

Es importante que la calibración sea realizada por personal competente, siguiendo procedimientos adecuados, preferiblemente aquellos que están basados en normas internacionales, como la ISO 376 (2004) o ASTM E74 (2009) y utilizando patrones adecuados, por ejemplo celdas de carga específicas para calibración o máquinas de calibración de masas suspendidas.

Los resultados de la calibración son el indicativo del funcionamiento de la nueva celda de carga. Dependiendo de los resultados, se puede determinar si la celda cumple con los requerimientos para el fin propuesto, que en este caso es la comprobación de máquinas de ensayo.

En términos generales, se puede decir que la celda de carga funciona bien para trabajo o para comprobaciones intermedias, si en los resultados de su calibración se reportan errores inferiores al $1 \%$ de la lectura, con una incertidumbre expandida $(U)$ también razonablemente baja, la cual podría rondar alrededor de $0,5 \%$ de la lectura (Tioli, 2007).

En la tabla siguiente se presenta el resumen de una calibración realizada a una celda de carga con una capacidad de $500 \mathrm{kN}$, construida para realizar comprobaciones intermedias de una máquina de ensayos universal de la misma capacidad.

La calibración de la celda de carga se realizó en el LanammeUCR, con patrones de fuerza adecuados para esta labor. En la Figura 12 se muestra la calibración de esta celda de $500 \mathrm{kN}$ de capacidad, utilizando una máquina de transferencia de fuerza.

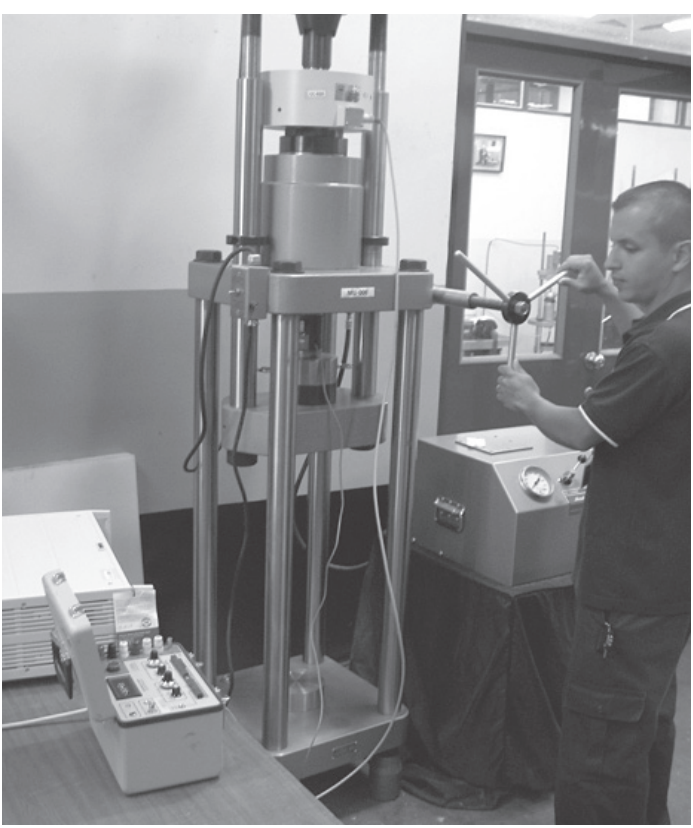

Figura 12. Tomada durante la calibración de la celda. Fuente: Los autores. 
Como se puede observar en la tabla anterior, los errores de indicación son todos inferiores al $1 \%$ de la lectura. Esto es un indicativo de que la celda de carga funciona satisfactoriamente y es adecuada para comprobaciones intermedias de máquina de ensayo. Adicionalmente, los valores de las incertidumbres reportadas son razonablemente bajos.

Es importante recalibrar la celda de carga de forma periódica. Como punto de partida, se recomienda calibrar la celda de carga cada año. Sin embargo, de acuerdo con el comportamiento demostrado a través del tiempo, este intervalo se puede aumentar o disminuir de acuerdo con la frecuencia de uso y del historial de resultados de calibraciones.

\section{CONCLUSIONES}

Es posible diseñar y construir celdas de carga a la medida y de bajo costo, apropiadas para la comprobación de máquinas de fuerza en laboratorios de ensayo. La metodología para el diseño puede ser aplicada por cualquier estudiante o profesional en ingeniería con conocimientos básicos de mecánica de materiales. La construcción de la celda se puede realizar en talleres de mecánica de precisión. La instrumentación requiere de habilidad para la colocación de galgas extensométricas.

Adicionalmente, en el país existe la disponibilidad del servicio de calibración de instrumentos de fuerza, lo cual es de gran ayuda para determinar si las celdas de carga construidas cumplen con los requisitos mínimos de precisión, exactitud e incertidumbres para los fines propuestos.

Una sola unidad lectora sirve para "leer" varias celdas de carga. Por lo tanto, se pueden construir celdas de varias capacidades que cubran todas las necesidades de un laboratorio.

Lo anterior permite que un laboratorio con una inversión relativamente baja pueda tener sus propias celdas de carga para comprobación. Estas celdas constituyen valiosas herramientas para el control metrológico de los equipos de fuerza de los laboratorios de materiales de construcción para obra

Cuadro 2. Resultados de la calibración de una celda de carga de $500 \mathrm{kN}$, diseñada y fabricada mediante el procedimiento expuesto en este documento

\begin{tabular}{|c|c|c|c|c|c|}
\hline \multicolumn{2}{|c|}{$\begin{array}{c}\text { Fuerza } \\
\text { indicada }(\mathbf{F i})\end{array}$} & \multicolumn{2}{|c|}{$\begin{array}{c}\text { Lectura fuerza } \\
\text { referencia promedio }\end{array}$} & \multirow{2}{*}{$\begin{array}{c}\text { U expandida } \\
\mathbf{k}=\mathbf{2} \\
+(\% \mathrm{~L})\end{array}$} & \multirow{2}{*}{$\begin{array}{c}\text { Error de } \\
\text { indicación } \\
\% \mathrm{~L}\end{array}$} \\
\hline$(\mathrm{kN})$ & (kgf) & $(\mathrm{kN})$ & (kgf) & & \\
\hline 49,03 & 5000 & 48,98 & 4994 & 0,454 & 0,11 \\
\hline 98,07 & 10000 & 97,83 & 9976 & 0,223 & 0,24 \\
\hline 147,10 & 15000 & 146,83 & 14973 & 0,168 & 0,18 \\
\hline 196,13 & 20000 & 195,77 & 19963 & 0,145 & 0,18 \\
\hline 245,17 & 25000 & 244,82 & 24965 & 0,163 & 0,14 \\
\hline 294,20 & 30000 & 293,88 & 29968 & 0,145 & 0,11 \\
\hline 343,23 & 35000 & 343,80 & 35057 & 0,411 & $-0,16$ \\
\hline 392,27 & 40000 & 393,95 & 40171 & 0,387 & $-0,43$ \\
\hline 441,30 & 45000 & 444,05 & 45280 & 0,372 & $-0,62$ \\
\hline 490,34 & 50000 & 494,09 & 50383 & 0,414 & $-0,76$ \\
\hline
\end{tabular}


civiles y el aseguramiento de la calidad de los resultados emitidos. En caso de que una celda de carga se dañe, puede ser reparada fácilmente, siguiendo la metodología expuesta en este documento.

El costo total de fabricación y calibración de la celda de carga es relativamente bajo, comparado con la compra de un instrumento de este tipo bajo las mismas condiciones, aparte de los beneficios y la confianza que se genera en el laboratorio con respecto a los resultados de los ensayos realizados.

\section{SIMBOLOGÍA}

kN kilonewton, unidad de fuerza del Sistema Internacional de unidades, múltiplo del newton

N newton, unidad de fuerza oficial del Sistema Internacional de Unidades

$\mathrm{kg}_{\mathrm{f}} \quad$ kilogramo fuerza, unidad de fuerza. Equi vale a 9,806 N.

$U_{\text {expandida }}$ incertidumbre expandida, usualmente con un factor de cobertura de $k=2$, para un nivel de confianza de aproximadamente $95 \%$.

$\% \mathrm{~L} \quad$ porcentaje de la lectura.

$\mu \mathrm{S}$ microstrains, deformación unitaria adimensional.

$\varepsilon \quad$ Deformación unitaria adimensional.

$\sigma \quad$ Esfuerzo dentro del rango elástico en megapascales (Mpa).

\section{REFERENCIAS BIBLIOGRÁFICAS}

American Society for Testing and Materials (ASTM). (2009). ASTM E74-09 Standard Practices for Force Verification of Testing Machines. West Conshohocken, PA.

BLH Electronics. SR-4 Strain Gage Handbook. Waltham, MA. Estados Unidos.

Holman, J. Métodos experimentales para ingenieros. México.

Harris, H. Structural Modeling and Experimental Techniques. EstadosUnidos
Measurements Group (1988). Strain Gage Based Transducers. Their design and construction. Raleigh. Estados Unidos.

Tioli, H. (2007). Diseño y construcción de una celda de carga para compresión, con una capacidad de $500 \mathrm{kN}$, para la Escuela de Ingeniería Mecánica. Trabajo final de investigación aplicada sometido a la consideración de la Comisión del Programa de Estudios de posgrado en Ingeniería Mecánica para optar por el grado de Magister en Ingeniería Mecánica, Universidad de Costa Rica, San José.

Norma ISO 10012:2003(E) Sistema de gestión de mediciones-Requisitos para procesos de medición y equipos de medición.

Norma ISO 376:2004(E) Metallic materialsCalibration of force-proving instruments used for the verification of uniaxial testing machines.

Instituto de Normas Técnicas de Costa Rica INTECO. INTE ISO/IEC 17025:2005 Requisitos generales para la competencia de los laboratorios de ensayo y de calibración. San José.

\section{SOBRE LOS AUTORES}

\section{Alejandro Navas}

Magister en Ingeniería Civil. Director del Laboratorio Nacional de Materiales y Modelos Estructurales de la Universidad de Costa Rica (LanammeUCR).

Teléfono: 2511-2508

Correo electrónico: alejandro.navas@ucr.ac.cr

\section{Humberto Tioli}

Magister en Ingeniería Mecánica. Gestor de Calidad y Jefe del Laboratorio de Fuerza del Laboratorio Nacional de Materiales y Modelos Estructurales de la Universidad de Costa Rica (LanammeUCR).

Teléfono: 2511-2514

Correo electrónico: jose.tioli@ucr.ac.cr 